\section{Robust Controller Design for a Series Resonant Converter}

\author{
ZI-QIN WANG \\ MARIO SZNAIER, Member, IEEE \\ Pennsylvania State University \\ ISSA BATARSEH, Senior Member, IEEE \\ University of Central Florida \\ JUANYU BU \\ Pennsylvania State University
}

\begin{abstract}
Because of their reduced switching losses, allowing a higher operating frequency, dc-to-dc resonant converters have been used extensively in the design of smaller size and lighter weight power supplies. The steady state and dynamic behavior of both the conventional series and parallel resonant converters have been thoroughly analyzed and small-signal models around given nominal operating points have been obtained. These models have been used in the past to design controllers that attempted to keep the output voltage constant in the presence of input perturbations. However, these controllers did not take into account either load or components variations, and this could lead to instability in the face of component or load changes. Moreover, prediction of the frequency range for stability was done a posteriori, either experimentally or by a trial and error approach.

In this paper we use $\mu$-synthesis to design a robust controller for a series resonant converter (SRC). In addition to robust stability the design objectives include rejection of disturbances at the converter input while keeping the control input and the settling time within values compatible with a practical implementation.
\end{abstract}

Manuscript received October 18, 1993; revised May 23, 1994 and March 10, 1995.

IEEE Log No. T-AES/32/1/00777.

This work was supported in part by NSF under Grant ECS-9211169.

Authors' addresses: Z. Q. Wang, M. Sznaier and J. Bu, Dept. of Electrical and Computer Engineering, Pennsylvania State University, University Park, PA 16802-2705; I. Batarseh, Dept. of Electrical Engineering, University of Central Florida, Orlando, FL 32816-2450.

$\overline{0018-9251 / 96 / \$ 10.00 ~(c) ~} 1996$ IEEE

\section{INTRODUCTION}

For many years, a conventional approach to control power conversion was to use switching mode power processing circuits based upon pulsewidth modulation (PWM) control methods [18]. In PWM converters, switching of semiconductor power devices is done under high current levels. Hence, in order to reduce switching losses, the frequency of operation of these converters is limited. In addition, the high frequency harmonic components resulting from quasi-square switching current and/or voltage waveforms produces high levels of electromagnetic interference (EMI) $[3,10]$.

In resonant converters, the capacitor voltage and current waveforms exhibit sinusoidal behavior, allowing for the use of high operating frequencies. These high frequencies are desirable since they result in smaller, lighter magnetic components and faster transient responses. In fact, today's dc-to-dc resonant converters have their operating frequencies well in the megahertz range [12], with power density up to $50 \mathrm{~W} / \mathrm{in}^{3}$. An additional advantage of resonant converters over PWM converters is a substantially lower harmonic content.

Due to these features and the ever increasing demand for smaller size and lighter weight high performance dc-to-dc converters for industrial, residential, and aerospace applications, high frequency dc-to-dc resonant converters are currently the object of widespread interest. Depending on the manner in which energy is transformed from the resonant tank to the output circuit, traditionally converters are classified as series $[4,13,19,21,28,31,34]$ and parallel $[2,4$, $11,14,19,20,31,33,34]$ resonant converters. The former type uses the inductor current to couple the energy from the resonant tank to the load, whereas the later type uses the capacitor voltage for the transfer of energy. In both types, the resonant tank $(L C)$ plays the role of buffering the energy from the source to the output circuit. The control characteristics of the series resonant converter reveal that for a small load variation, a wide range of switching frequencies is required. Hence, it is used in applications with tight load regulations [13, 20,28]. Unlike the series types, the parallel resonant converter is more suited to applications requiring a wide range of load variations $[11,14,33]$.

The steady state and dynamic behaviors of both the conventional series and parallel resonant converters have been thoroughly analyzed. Several commonly used control techniques were compared in [22]. As shown there, most of these techniques have relatively poor performance. The alternative control law proposed, the optimal trajectory control method, achieves good nominal performance, but it entails using a complex, nonlinear controller. More recently small signal models have been obtained using perturbation methods, and 


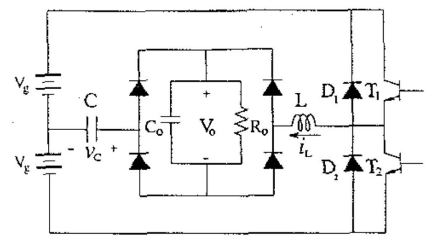

Fig. 1. Conventional SRC circuit diagram.

used in the design of controllers that attempt to keep the output voltage constant in the presence of input perturbations [29]. However, these controllers did not take into account either load or components variations. Moreover, prediction of the frequency range for stability was done a posteriori, most of the time either experimentally or by a trial and error approach.

We use $\mu$-synthesis (see [23, 24] and references therein) to design a robust controller for a series resonant converter (SRC). The design objective is to robustly reject input variations in the presence of load and component uncertainty, while keeping both small control actions and settling times. This is accomplished by selecting appropriate weight functions reflecting these requirements.

This work is organized as follows. In Section II we briefly describe the conventional SRC and we provide a small signal model around a nominal operating point. In Section III we analyze the characteristics of the plant. This analysis provides some insight into the nature of the control problem, in particular displaying the relatively poor control characteristics of the plant. In Section IV we indicate how to characterize plant uncertainty and we design a robust controller to achieve robust performance (i.e. guaranteed performance for all possible plants) using $\mu$-synthesis. In Section V we provide linear and nonlinear simulation results showing the performance of the closed-loop system under different conditions. Finally, in Section VI we summarize our results.

\section{PROBLEM DESCRIPTION}

\section{A. The Conventional Series Resonant Converter}

The conventional SRC circuit diagram [29] is shown in Fig. 1. The series resonant circuit consists of the inductor $L$ and the capacitor $C$. The parallel combinations of the transistors and the diodes form bidirectional switches which operate at fifty percent duty ratio to generate a symmetrical square wave voltage, with frequency $f_{s}$, applied across the resonant circuit. The resonant inductor current is coupled to the output circuit using a full wave rectifier. The output capacitor $C_{o}$ behaves as a constant voltage sink in the steady state and is much larger than the resonant capacitor $C$. Finally, the resistor $R_{o}$ and the voltages
$V_{g}$ and $V_{o}$ represent the load, the line (input) and the output, respectively.

The nominal parameters used here are the same as the data in [29], i.e.,

$L=0.1335 \mathrm{mH}$

$C=12.8 \mathrm{nF}$

$C_{o}=32 \mu \mathrm{F}$

$R_{o}=19 \Omega$

$V_{g}=40 \mathrm{~V}$

$V_{o}^{g}=15 \mathrm{~V}$

$f_{s}=100.35 \mathrm{kHz}$.

For convenience, we introduce the following normalized variables:

$V_{n g}=V_{g} / V_{g}=1$

$V_{n o}=V_{o} / V_{g}=0.375$

$V_{n c}=V_{c} / V_{g}$

$I_{n l}=Z_{o} I_{l} / V_{g}=\sqrt{L / C} I_{l} / V_{g}$

$F_{n s}=f_{s} / f_{o}$.

where $f_{o}=1 / 2 \pi \sqrt{L C}$ is the resonant frequency. It should be noted that the normalized output $V_{n o}$ is the same as the converter gain. Finally, the load condition is defined as: $Q=\sqrt{L / C} / R_{o}$.

\section{B. Small Signal Model}

Under steady-state conditions, it can be shown that, for a SRC operating in the continuous conduction mode [29], there are four circuit modes in each switching period. Thus the converter is a nonlinear, variable structure system, with its steady state state-trajectory uniquely determined by the normalized switching frequency $F_{n s}$ and load condition $Q$ [28, 34]. For a given operating point, a small signal discretetime model of the converter can be obtained by using a perturbation method [29]. The sampling time for this discrete time model is equal to $T_{s} / 2$, where $T_{s}=1 / f_{s}$ is the switching period. Therefore, due to aliasing effects, this model is accurate up to the operating switching frequency.

The discrete model from the the normalized switching frequency $F_{n s}$ and the normalized line $V_{n g}$ to the normalized output $V_{n o}$ (to simplify the notation, we use the same variables for both the steady state and its perturbation) at the nominal operating point is given by the following state space realization [29]:

$$
\begin{aligned}
x(k+1) & =A x(k)+B\left(\begin{array}{c}
F_{n s}(k) \\
V_{n g}(k)
\end{array}\right) \\
V_{n o}(k) & =C x(k)
\end{aligned}
$$

where

$$
\begin{aligned}
x^{T} & =\left(\begin{array}{lll}
V_{n c} & I_{n l} & V_{n o}
\end{array}\right) \\
A & =\left(\begin{array}{rrr}
0.7107 & 0.9449 & -2.5271 \\
-0.4335 & 0.6742 & -0.4008 \\
0.0006 & 0.0011 & 0.9905
\end{array}\right)
\end{aligned}
$$




$$
\begin{aligned}
B & =\left(\begin{array}{rr}
-4.1482 & 0.6637 \\
10.6373 & 1.6742 \\
-0.0005 & 0.0008
\end{array}\right) \\
C & =\left(\begin{array}{lll}
0 & 0 & 1
\end{array}\right) .
\end{aligned}
$$

\section{Control Objectives}

The purpose of the feedback controller is to keep the output voltage at a prescribed level (in our case $V_{o}=15 \mathrm{~V}$, i.e., $V_{n o}=0.375$ ) at all operating points, using as control input the switching frequency $f_{s}$. This problem can further be divided into three parts as follows.

1) Line Regulation (Nominal Performance): The line voltage is often unregulated and could have a substantial range of variation, with typical variation values around $\pm 20 \%$. This variation is modeled as an external disturbance, thus leading to a disturbance rejection problem. Commonly used performance specifications for this disturbance rejection problem are a) zero steady state error; b) output overshoot less than 10\%; and c) settling time of at most $5 \mathrm{~ms}$.

2) Load Regulation (Robust Stability): On the other hand, the load condition could also vary over a wide range. Since the load $R_{o}$ enters the dynamics of the model, load variations will appear as model uncertainty and could possibly lead to stability problems. Normally the load changes from $10 \%$ at low load to $90 \%$ at full load condition. Other model uncertainty, such as unmodelled high frequency dynamics, are also considered.

3) Robust Performance: Since the converter operates at a wide range of load condition, the performance requirements must be satisfied at all operating points. This is equivalent to requiring satisfactory response under both line and load variations.

4) Finally, in order to guarantee implementability of the resulting controller, all physical variables such as control input must be limited to practical values.

REMARK 1 Note that robust performance is achieved as long as the minimum performance requirements are met by all possible plants, (i.e., the worst case performance still satisfies the specifications), even though the responses corresponding to different plants may differ significantly due to the wide variation of operating conditions. A related problem is to design a controller that renders the performance of the plant insensitive to model uncertainty. Although this will result in closer responses for all plants in the set, it will also usually deteriorate performance.

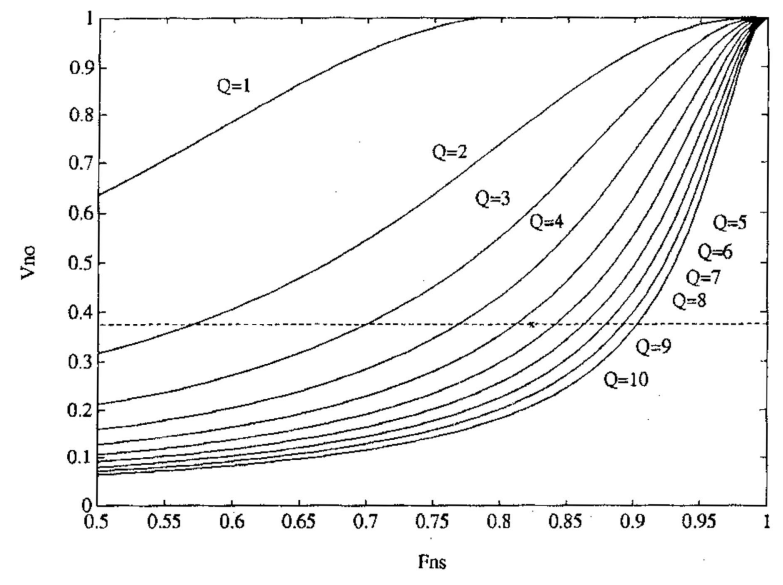

Fig. 2. Conventional SRC control characteristics curves.

\section{ANALYSIS OF THE PLANT}

To gain an essential understanding of the problem, we proceed with an analysis of the plant before designing a controller.

\section{A. Control Characteristics}

In steady state, if any two of the variables among the normalized output $V_{n o}$, switching frequency ratio $F_{n s}$, and output load $Q$ are specified, the third variable can be determined. The effects of the switching frequency and the load upon the converter output can be easily visualized by using the control characteristic curves. From the control point of view, the control characteristic curves allow us to make a initial estimate of the load change that can be tolerated and to see some of the difficulties in load regulation.

The control characteristic curves for various output loads $Q$ are shown in Fig. 2. As pointed out in [29], we see that the SRC has a relatively poor control characteristics. Since different control characteristics curves are far apart, a wide range of switching frequencies is needed to accommodate load changes. This further implies that the plant dynamics may vary significantly as the operating point changes. Here, the operating point varies along the dotted line in Fig. 2 where the mark $\mathbf{x}$ indicates the nominal operating point.

\section{B. Frequency Responses}

From the discrete time state space model, we can easily get the $z$-transfer functions from the normalized switching frequency and the normalized line input to the normalized output:

$$
\left[G(z) G_{g}(z)\right]=C(z I-A)^{-1} B .
$$

Following a common approach, we carry out the analysis of the plant and the synthesis of a digital controller using a $w$-plane approach [9]. To this effect, 
the bilinear transformation:

$$
z=\frac{1+s T_{s} / 4}{1-s T_{s} / 4}
$$

is used to get the transfer functions in the frequency domain $s$. These transfer functions, still denoted as $G$ and $G_{g}$, are given by

$$
\begin{aligned}
G(s) & =\frac{1.545 * 10^{-3}(s+447980)(s-362050)(s-401400)}{(s+1747.4)(s+13443-157260 i)(s+13443+157260 i)} \\
G_{g}(s) & \left.=\frac{8.207 * 10^{-5}(s+267090)(s-1874400)(s-401400)}{(s+1747.4)(s+13443-157260 i)(s+13443+15726} i\right)
\end{aligned}
$$

The frequency responses $G(s)$ corresponding to the nominal operating point as well as to a few other load conditions are shown in Fig. 3. At the nominal operating point, the converter has one real pole at -1747.4 and a pair of conjugate poles at $-13443 \pm 157260 i$, responsible for the peak in the frequency response. As the load becomes lighter, the conjugate poles move farther to the left, with the peak decreasing, until they change into two real poles. If the load is decreased further, one of the real poles moves very fast towards $-\infty$ and then at $R_{o}=47.1 \Omega$ from $-\infty$ to $+\infty$, yielding an unstable open-loop plant. At this point, the perturbation technique used to obtain the small-signal model ceases to be valid. Therefore, in this paper, we limit $R_{o}$ to be less than $45 \Omega$. On the other hand when the load becomes heavier, the conjugate poles move towards the imaginary axis, resulting in a larger resonant peak. As we explain in detail later, this results in a more difficult control problem.

\section{CONTROL DESIGN}

\section{A. Structured Singular Value and $\mu$-Synthesis}

Consider the system interconnection shown in Fig. 4(a), where $M$ represents the nominal (stable) closed-loop system and where the "feedback" $\Delta_{u}$ represents a model perturbation with a block diagonal structure of the form: (see [7])

$$
\begin{aligned}
\Delta_{u}(s) & \in \boldsymbol{\Delta} \\
& \doteq\left\{\Delta(s)=\text { block }-\operatorname{diag}\left\{\Delta_{1}(s), \Delta_{2}(s), \ldots, \Delta_{n}(s)\right\},\right. \\
& \left.\Delta_{i}(s) \text { stable }\right\} .
\end{aligned}
$$

This interconnection can be used to represent a nominal system subject to different types of model uncertainty. The stability of this interconnection has been analyzed in [7, 25, 27]. In [25, 27], Safonov and Athans defined the multivariable stability margin $k_{m}$ as

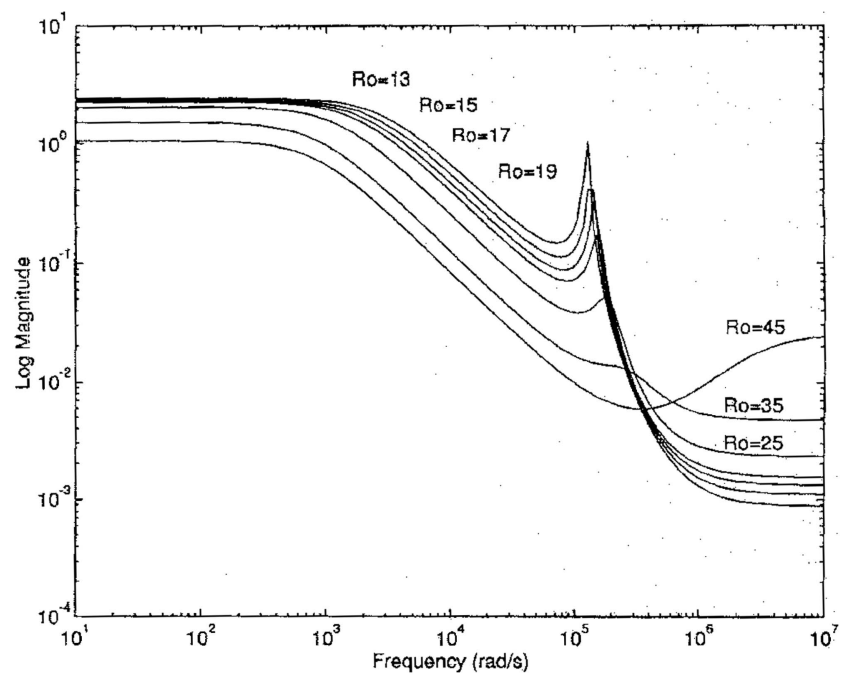

Fig. 3. Frequency responses $G(s)$ at different load conditions.

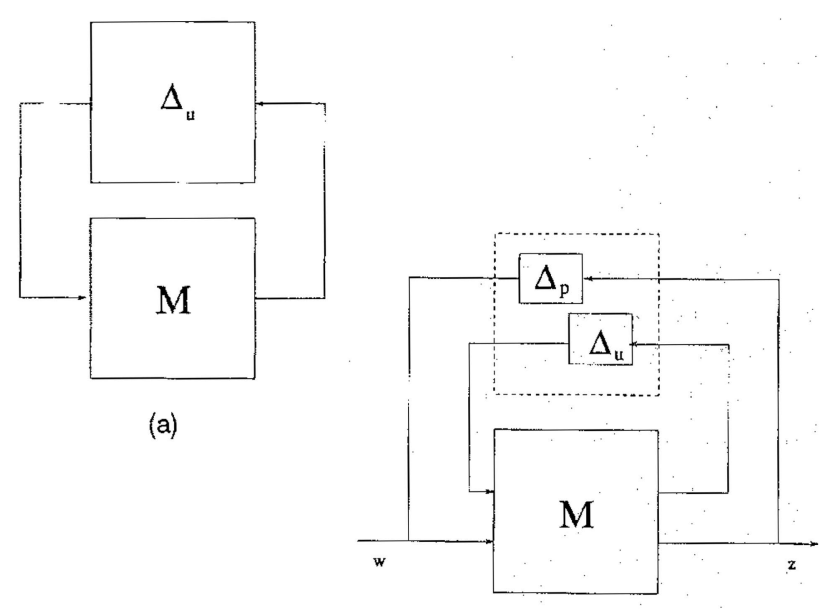

(b)

Fig. 4. (a) Robust stability problem. (b) Robust performance as robust stability problem.

the largest positive $k_{m}$ such that the interconnection is stable for all $\Delta,\|\Delta\|_{\infty} \leq k_{m}$, i.e.,

$$
\begin{gathered}
k_{m_{\Delta_{u}}}(M)=\min _{\Delta_{u} \in \Delta}\left\{\left\|\Delta_{u}\right\|_{\infty}: \operatorname{det}\left(I+M(j \omega) \Delta_{u}(j \omega)\right)\right. \\
=0 \text { for some } \omega\}
\end{gathered}
$$

where $\left\|\Delta_{u}\right\|_{\infty} \doteq \sup _{w} \bar{\sigma}\left(\Delta_{u}\right)$ and where $\bar{\sigma}(\cdot)$ denotes the maximum singular value. Thus, $k_{m}$ is an indicator of the the largest uncertainty permissible before instability occurs. In [7], Doyle introduced the concept of Structured Singular Value (SSV or $\mu$ ), defined as

$$
\mu_{\Delta_{u}}(M)=\left\{\begin{array}{c}
\frac{1}{\min _{\Delta_{u} \in \Delta}\left\{\left\|\Delta_{u}\right\|_{\infty}: \operatorname{det}\left(I+M(j \omega) \Delta_{u}(j \omega)\right)\right.} \\
=0 \text { for some } \omega\} \\
0 \quad \text { if no } \Delta_{u} \in \Delta \text { destabilizes } M
\end{array} .\right.
$$

Hence, $\mu$ is simply equal to the reciprocal of $k_{m}$. 
As shown in [7], if $M$ is a stable transfer matrix, a necessary and sufficient condition for robust stability of the interconnected system for all perturbations $\left\|\Delta_{u}\right\|_{\infty} \leq 1$ is that

$$
\mu_{\Delta_{u}}(M)<1 .
$$

Robust performance (i.e. guaranteed performance for all possible plants in the set) can be addressed by recasting the problem into an augmented robust stability problem by introducing an additional fictitious perturbation block $\Delta_{p}$, as shown in Fig. 4(b), where $w$ and $z$ represent exogenous inputs and outputs subject to performance specifications, respectively. It can be shown (see the main loop theorem in [24]) that robust performance is achieved if and only if:

$$
\mu_{\Delta}(M)<1
$$

where $\Delta=\operatorname{diag}\left\{\Delta_{p}, \Delta_{u}\right\}$ contains now both the uncertainty and the performance blocks.

As shown above, $\mu$ provides a useful tool for robustness analysis. It combines unstructured and structured uncertainty, robust stability and robust performance in a unified framework. It can even be extended to cover parametric uncertainty (real $\mu$ ). Unfortunately, at the present time there are no efficient algorithms for computing the exact value of $\mu$ for general perturbation structures. Thus, the following upper bound is used instead [7, 27]:

$$
\left.\mu_{\Delta}(M) \leq \inf _{D \in \mathcal{D}} \| D M D^{-1}\right) \|_{\infty}
$$

where $\mathcal{D}$ represents a set of positive definite Hermitian matrices with a diagonal block structure that commute with $\Delta$. Efficient computational algorithms exist for this upper bound. Moreover, this upper bound coincides with the exact value for perturbation structures having up to 3 blocks. For more than 3 blocks, the bound is no longer tight. However, the largest gap ever observed is less than $15 \%$ (corresponding to an example built analytically), and is substantially lower in most cases arising in practice [24].

From the discussion above it follows that robust controllers guaranteeing robust stability or robust performance can be synthesized by solving the following optimization problem:

$$
\min _{K \text { stabilizing }} \mu_{\Delta}\{M(K)\}
$$

where the notation $M(K)$ is used to indicate explicitly that the closed-loop transfer matrix $M$ is a function of the controller $K$. Due to the difficulties in computing the exact value of $\mu$, the upper bound (8) is used instead, yielding the following optimization problem:

$$
J=\min _{K}\left\{\inf _{D \in \mathcal{D}}\left\|D M(K) D^{-1}\right\|_{\infty}\right\} .
$$

Robust stability or robust performance is achieved if $J<1$. The optimization problem (9) is convex both in the scaling $D$ and in the controller $K$. However, it is not jointly convex and thus hard to solve (due to the possible presence of multiple local minima). The solution method currently used alternates between finding the tightest possible upper bound by optimizing the scales $D$ while holding the controller constant (an infinite dimensional convex optimization problem); and finding an internally stabilizing controller that minimizes this upper bound (a standard $\mathcal{H}_{\infty}$ control problem). This algorithm, known as the " $D-K$ " iteration, is implemented both in the Robust Control Toolbox [1] and $\mu$ Analysis and Synthesis Toolbox [6], and can be summarized as follows.

1) $\mathcal{H}_{\infty}$ Synthesis. Holding $D$ fixed, use $\mathcal{H}_{\infty}$ synthesis to solve:

$$
\min _{K}\left\|D M(K) D^{-1}\right\|_{\infty} .
$$

In the first iteration $D$ is often set to $I$. After the first iteration the $D$ scale obtained in Step 3 is used.

2) $\mu$ Analysis. Calculate the upper bound of $\mu$ for the closed-loop system obtained using the controller $K$ from Step 1. This entails solving the following infinite-dimensional optimization problem:

$$
\inf _{D \in \mathcal{D}}\left\|D M(K) D^{-1}\right\|_{\infty} \text {. }
$$

This problem is approximately solved by finding the value of $D$ over a finite grid of frequency points $\omega_{i}$.

3) $D$ Fitting. The approximate solution to the optimal scaling problem of Step 2 is found by fitting the values $D\left(j \omega_{i}\right)$ with a real-rational, proper, stable, minimum-phase transfer matrix $D(s)$.

4) Go to Step 1 if stop criterion is not met.

Due to the lack of joint convexity in $K$ and $D$, this algorithm is not theoretically guaranteed to converge to the global minimum. An additional drawback is that the user has to make a judgement call in Step 3, when deciding the order of the rational function $D(s)$ used to interpolate the data points, and different choices may yield widely different results. In practice however, the $D-K$ iteration seems to work well, and has allowed for solving many difficult engineering problems (see for instance [30]). Interested readers are referred to [23, 24] for a more detailed discussion of the history and theory of both $\mu$ and $\mu$-synthesis.

\section{B. Uncertainty Weight Selection}

In this work we describe the set of possible plants using multiplicative dynamic uncertainty, i.e., we assume that the actual plant can be modeled as

$$
G(s)=G_{o}(s)(1+W(s) \Delta(s))
$$

where $G_{o}(s)$ represents the nominal plant, $W(s)$ is a fixed weighting function that contains all the information available about the frequency distribution of the uncertainty, and where $\Delta(s)$, a stable transfer 


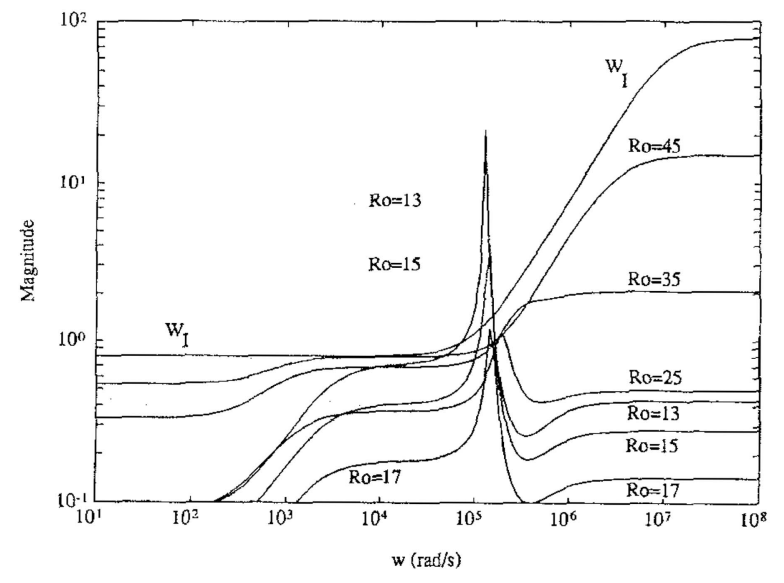

Fig. 5. Multiplicative uncertainty for different loads $R_{o}$ and uncertainty weights.

function, represents model uncertainty. Furthermore, we assume, by absorbing any scaling factor into $W(s)$ if necessary, that $\|\Delta(s)\|_{\infty} \leq 1$. Thus, at each frequency $\omega$, all possible plants lie in the disk centered at the nominal frequency response $G_{o}(\omega)$, with radius $r(\omega)=$ $\left|G_{o}(j \omega) W(j \omega)\right|$ (hence this uncertainty description is sometimes referred as disk-like uncertainty).

There are a number of uncertainty sources in the converter modeling. Load variation is, of course, a primary source. In this work we assume that $R_{o}$ is within the range from $17 \Omega$ to $45 \Omega$ which corresponds to $Q$ within the range from 2.2695 to 6.0074 . The nominal operating point corresponds to $R_{o}=19 \Omega$, with the corresponding $Q=5.375$. Additionally, there might be some uncertainties in the resonant inductor $L$ and capacitor $C$. Since they result in a change of the load condition $Q$, they effectively behave as a load resistance $R_{o}$ change. Hence we do not consider these uncertainties separately. Other uncertainty sources are neglected high frequency dynamics and limitation of the small-signal modeling approach. Since these two are primarily high frequency uncertainties, it follows that the uncertainty weight needs to be sufficiently large at high frequencies.

In this work we address this model uncertainty by using a single, norm bounded, multiplicative uncertainty to cover all possible plants. Let $G_{o}(s)$ represent the nominal transfer function from the control input $F_{n s}$ to the output $V_{n o}$, and let $G^{R_{o}}(s)$ denote the transfer functions for operating points where $R_{O}$ is different from its nominal value. These off-nominal plants can be represented by the model (12) by defining:

$$
\begin{aligned}
\Delta^{R_{o}}(w) & =\left(G^{R_{o}}-G\right) G^{-1} \\
r^{R_{o}}(\omega) & =\left|\Delta^{R_{o}}(\omega)\right|=\left|\left(G^{R_{o}}-G\right) G^{-1}\right| .
\end{aligned}
$$

Some sample uncertainties corresponding to different values of the load $R_{o}$ are shown in Fig. 5. We can see that the multiplicative uncertainties have a peak at the oscillating frequency. This peak becomes larger and steeper as the load resistance $R_{o}$ decreases. Covering this steep peak by a simple rational uncertainty weight seems very hard, hence it inevitably introduces much conservatism to get a reasonable simple uncertainty weight. This explains partially why in the electronics community, the SRC control systems are designed on the heaviest load.

Based on the above discussion, the following multiplicative uncertainty weight was chosen for control design:

$$
w_{I}(s)=0.8 \frac{10^{-5} s+1}{10^{-7} s+1} .
$$

The magnitude frequency response of $w_{I}(s)$ is also shown in Fig. 5. It begins to increase at the frequency $\omega=10^{5} \mathrm{rad} / \mathrm{s}$ and does not stop until reaching 80 at the frequency $\omega=10^{7} \mathrm{rad} / \mathrm{s}$, thus covering both, high frequency uncertainties and all the uncertainties due to load changes from $17 \Omega$ to $45 \Omega$. Attempting to cover the resonant peak corresponding to values of $R_{o}<17$ with a low order rational function results in a larger gap between the sets of plants and the uncertainty used to cover it both at low and high frequencies. Thus, this approach introduces too much conservatism, deteriorating the performance. On the other hand, a tighter fit could be achieved by using a higher order function to model the uncertainty. However, this results in a high-order controller. Thus the weight (15) offers a good compromise between robustness and controller complexity.

The relatively large magnitude of (15) at low frequencies $(0.8)$ is due to the wide range of operating points, which results in a significant change in static gain. It should be pointed out that static gain change never leads to disk-like uncertainty of the form (12) at low frequencies because phase does not change much at low frequencies. Hence, this approach is potentially conservative since it allows for uncertainties than never appear in practice. To exclude these unexisting uncertainties, real $\mu$ may be used. However, as shown in [35], robustness depends only on the worst case uncertainty, introducing these additional uncertainties will not yield a (much) more conservative design as far as these additional uncertainties do not affect (much) the original worst case uncertainty. Uncertainty modeling can be improved by using the following uncertainty weight

$$
w_{I}^{\prime}(s)=0.5 \frac{(0.016 s+1)\left(10^{-5} s+1\right)}{(0.001 s+1)\left(10^{-7} s+1\right)}
$$

which has the same magnitude as $w_{I}(s)$ at middle and high frequencies but reduces the magnitude from 0.8 to 0.5 at low frequencies. However we found that this improved modeling made little difference in the performance of the closed-loop system.

The uncertainty in the feedforward path $G_{g}(s)$ can be modeled in the same way. However, following 


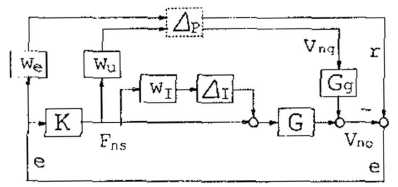

Fig. 6. Block diagram for $\mu$-synthesis.

a standard practice, we have not included this uncertainty explicitly in the control design. The reasons are as follows. 1) An additional perturbation has to be used to represent this uncertainty. However, this perturbation is neither totally independent nor identical to the uncertainty in feedback loop, as both of them result from the same source-load variation. Thus, adding this second perturbation not only complicates the problem, but it also potentially introduces much conservativeness. 2) This feedforward path uncertainty does not affect robust stability. 3) As we show in the next section, this uncertainty is taken into account when imposing robust performance requirements.

\section{Performance Weight Selection} where the uncertainty in the feedforward transfer function $G_{g}$ has been absorbed into the performance block $\Delta_{p}$. Here $\Delta_{I}$ and $w_{I}$ represent the model uncertainty perturbation block and its weight, respectively, which have been discussed in the previous subsection. $\Delta_{p}, w_{e}(s)$, and $w_{u}(s)$ represent the fictitious uncertainty block associated with the performance specifications and the performance weights associated with the tracking/regulation error and the control effort, respectively. Although regulation is our primary concern, the reference input $r$ is also included in our problem setting, in order to get good tracking characteristics.

The selection of $w_{e}(s)$ and $w_{u}(s)$ entails a trade-off among different performance requirements, particularly good regulation versus peak control action. The weight on the control error $w_{e}(s)$ is usually selected to be very large at low frequencies in order to get good tracking and regulation. One simple form is

$$
w_{e}(s)=\frac{\frac{T s}{M}+1}{T s+A}
$$

where $A$ is the maximum allowed steady state error ( $A$ should be zero if zero steady state is required); $T$ approximately determines the bandwidth $\left(\omega_{b} \approx 1 / T\right)$ and hence the rising time and settling time; and where $M$ is the maximum allowed amplification of high frequency noise. $M$ is closely related to the overshoot in the time response, and a common rule of thumb is to choose $M$ between 1.5 to 2 . The weight on the control input $w_{u}(s)$ was chosen close to a differentiator to penalize fast changes and large overshoot in control input. Interested readers may refer to [16] for more details.

Note that there is no exact relationship between the the parameters $T\left(\omega_{b}\right)$ and $M$ of the frequency domain weighting functions, and time domain performance specifications given in terms of rise-time, settling-time, and overshoot. The design of multiobjective robust controllers subject to both time and frequency domain specifications is to a large extent an open problem, although some progress has been made recently (see [32 and references therein]).

When using frequency domain weights to enforce time-domain specifications, the relationship for second-order systems presented in most classic control textbooks may be used for an initial weight selection. An iterative procedure, alternating between weight selection, controller synthesis and performance evaluation, is then usually needed in order to obtain a satisfactory design. When all the performance specifications are met but there is still room left for improvement, usually we only improve $T$, in order to get a response as fast as possible, while still satisfying the additional specifications.

The following weights offer a good compromise , among all the conflicting time-domain specifications:

$$
\begin{aligned}
& w_{e}(s)=\frac{0.001 s+1}{0.002 s} \\
& w_{u}(s)=\frac{5 * 10^{-5} s}{10^{-7} s+1} .
\end{aligned}
$$

These weights approximately give a closed-loop bandwidth of $1 / 0.002=500 \mathrm{rad} / \mathrm{s}$. Note that an integrator is included in $w_{e}(s)$ to get zero steady-state error. We may relax this requirement if we allow for a small, non-zero, steady-state error.

It should be pointed out that, in principle, the reference input $r$ and the weight on the control input $w_{u}(s)$ are not necessarily involved in the design since our main objective is line voltage regulation and since we did not impose specific requirements either upon the command tracking error, or upon the maximum control effort. If neither $r$ nor $w_{u}(s)$ are included in the problem setting shown in Fig. 6, then the following higher performance weight can be used:

$$
w_{e}^{\prime}(s)=\frac{1}{2} \frac{10^{-5} s+1}{10^{-5} s} .
$$

Using this weight robust performance $\left(\mu_{\mathrm{RP}}<1\right)$ can still be achieved, while obtaining acceptable control input under line voltage variations. However, the control input due to a reference input change has very large peaks, due to the presence of the transfer function $G_{g}(s)$ in the disturbance channel. Finally, it should be pointed out that the weight on the control input $w_{u}(s)$ alone has little effect, since the control action in response to a line voltage variation is acceptable. 


\section{Controller Synthesis}

By using the uncertainty description developed in Section IVB and the performance weights of Section IVC, we get an uncertainty structure $\Delta$ consisting of a scalar block (corresponding to the robust stability requirements) and a $2 \times 2$ block (corresponding to the robust performance). The robust controller was synthesized using the $\mu$ Analysis and Synthesis Toolbox [1], applied to the block diagram shown in Fig. 6. Initially the $D$ scale was set to $I$. After 5 iterations using a second-order fit for the $D$ scale, we obtained a 13th-order controller yielding $\mu_{\mathrm{RP}}=0.9823$. Finally, Hankel norm model reduction yielded a 6th-order controller with virtually no performance degradation $\left(\mu_{\mathrm{RP}}=0.9845<1\right)$. The state space description of this reduced order controller is given by

$$
K=C_{k}\left(s I-A_{k}\right)^{-1} B_{k}+D_{k}
$$
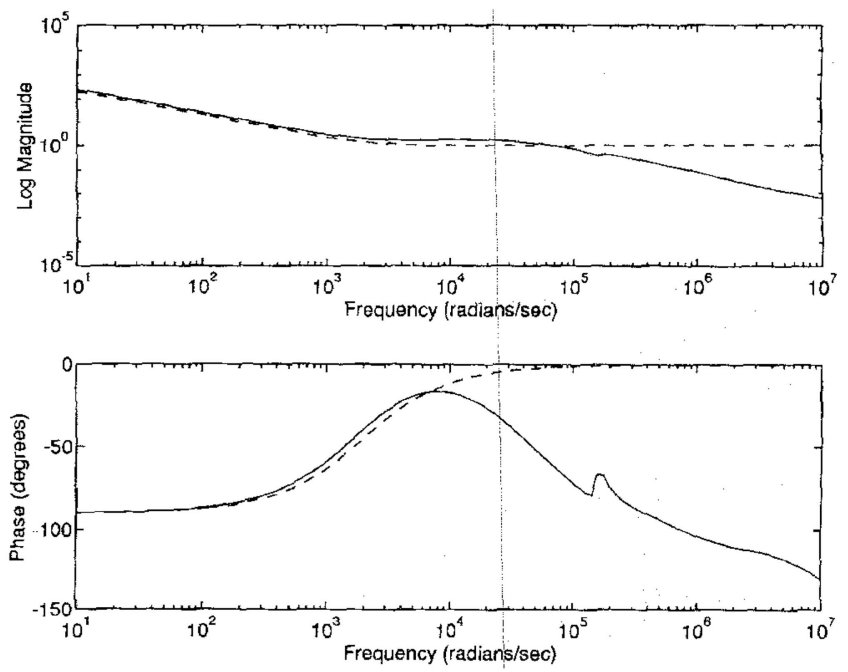

Fig. 7. Frequency responses of $\mu$ (solid) and PI (dash) controllers.

where

$$
\begin{aligned}
A_{k} & =\left(\begin{array}{cccccr}
-1.317 e+6 & 6.162 e+4 & 2.973 e+5 & 5.876 e-3 & -1.134 e+5 & -2.959 e+4 \\
0 & -1.552 e+3 & -1.667 e+5 & 4.899 e-4 & 1.420 e+4 & 4.090 e+3 \\
0 & 1.554 e+5 & -3.473 e+4 & -2.473 e-3 & 3.925 e+4 & 1.089 e+4 \\
0 & 0 & 0 & -1.000 e-3 & -5.265 e-3 & 3.900 e-4 \\
0 & 0 & 0 & 0 & -4.273 e+4 & -2.219 e+4 \\
0 & 0 & 0 & 0 & 0 & -7.641 e+3
\end{array}\right) \\
B_{k}^{T} & =\left(\begin{array}{llllll}
-153.51 & 38.575 & 56.637 & -50.427 & -278.14 & -49.935
\end{array}\right) \\
C_{k} & =\left(\begin{array}{llllll}
189.56 & 34.243 & 17.456 & -50.427 & -244.77 & -103.71
\end{array}\right) \\
D_{k} & =-3.8034 e-3 .
\end{aligned}
$$

Finally, the corresponding $z$-domain controller is obtained by using the inverse of the bilinear transformation (2).

In order to benchmark the performance of the robust controller, we also designed a proportional-integral-derivative (PID) controller. This controller, tuned based on the plant frequency responses at the various operating points shown in Fig. 3 , is given by

$$
K_{\mathrm{PI}}=\frac{s+2000}{s} .
$$

Notice that this is in fact a proportional-integral (PI) controller. The gain on the $D$ term was set to 0 to limit the gain at high frequency, since this gain has a direct bearing upon the robustness of the closed-loop gain. The frequency responses of both $\mu$ and the PI controllers are shown in Fig. 7. Both controllers have similar responses at low frequencies, with a slightly lower gain for the PI. Beyond $\omega=50000 \mathrm{rad} / \mathrm{s}$, the gain of the $\mu$ controller begins to decay very fast, in order to accommodate the model uncertainty at high frequencies. Note that robust stability dictates that the loop gain must be kept small enough at the resonant frequency. Since the response of the PI controller is flat at high frequency, the only mean to increase robustness is to reduce its gain.

\section{E. Controller Implementation Considerations}

Given the relatively high frequency sampling rate (roughly $200 \mathrm{Khz}$ ), of the plant, a digital implementation of the 6th-order $z$-domain controller may require using a specialized digital signal processing (DSP) processor, with enough processing power to carry out the required operations in the amount of time available. Rather than pursuing this approach, in this work we propose to implement this controller using an analog, continuous time controller, connected to the plant through the use of sample and hold 

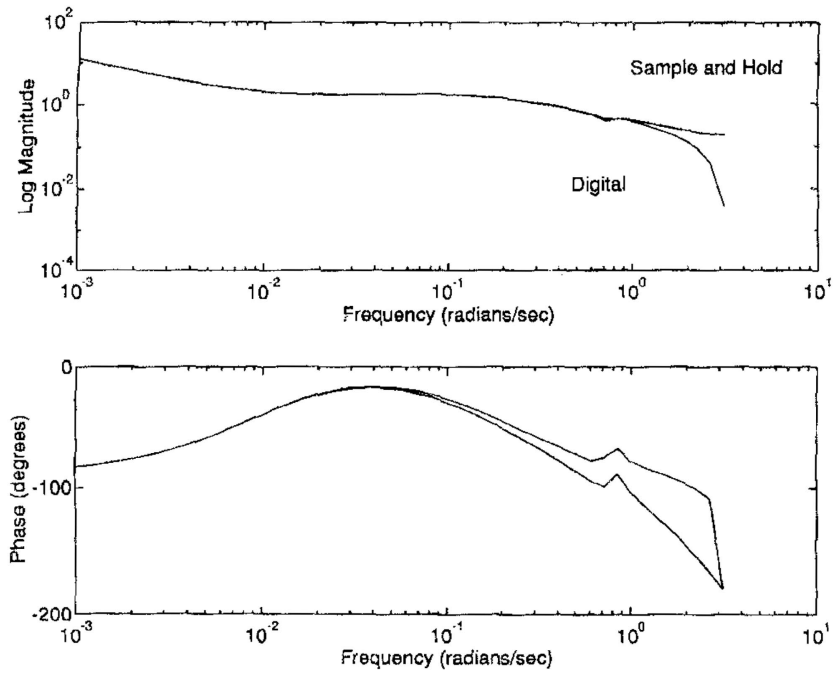

Fig. 8. Frequency responses of digital and sampled controllers.

devices. Fig. 8 shows the frequency response of the $\mu$-controller obtained by using the bilinear transformation to convert the $s$-domain controller (21) back to the $z$-domain, versus the frequency response of the $z$-domain controller obtained by simply sampling the inputs and outputs of (21). From the figure it follows that both responses are quite close, due to the sampling frequency being much higher than the controller bandwidth. Moreover, notice that the converter itself provides a sample and hold action when connected to the controller. Thus, connecting the $s$-domain controller (21) directly to the plant should provide a response closely resembling that of the true $z$-domain $\mu$-controller. This is the case, as we show in Section VB through the use of a nonlinear simulation of the closed-loop system.

\section{SIMULATION RESULTS}

\section{A. Linear Simulation}

The closed-loop system corresponding to the $\mu$-controller was simulated at four different operating points: $R_{o}=45 \Omega, 19 \Omega, 17 \Omega$, and $13 \Omega$, using the corresponding linear model of the plant. The time responses to $20 \%$ step change in line voltage $V_{n g}$ and reference input $r$ are shown in Fig. 9.

For the nominal case $R_{o}=19 \Omega$, the settling time is about $2 \mathrm{~ms}$ for line voltage change and is less than $1 \mathrm{~ms}$ for reference input change. The output responses are very good since the settling time is much less than the required $5 \mathrm{~ms}$, with almost no overshoot. The control action in response to a line voltage change is also adequate. However the control response to a reference input change has an overshoot. In our original design, without using a penalty weight $w_{u}(s)$ on the control, this peak value was almost 2 ,
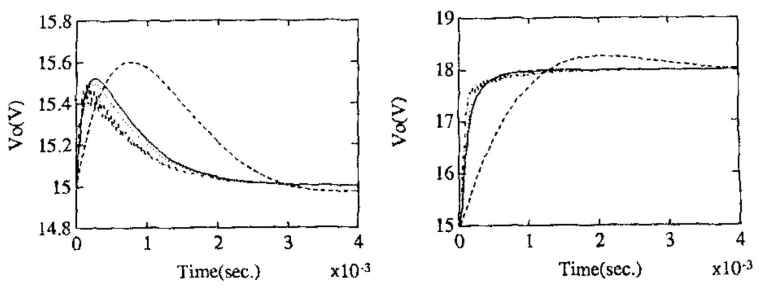

(a)
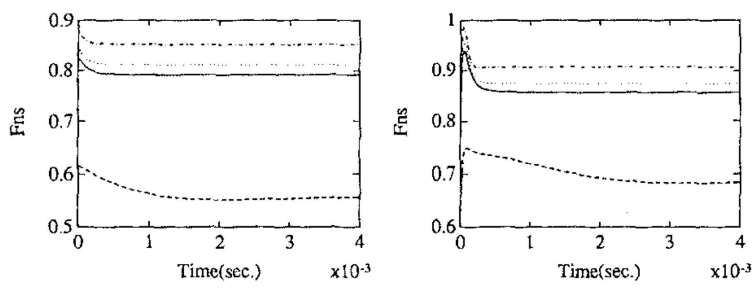

(b)

Fig. 9. Linear simulation results with $\mu$-controller at different operating points $R_{o}=19$ (solid), 45 (dash), 17 (dot), 13 (dot-dash) $\Omega$. (a) Line voltage step change (20\%). (b) Reference input step change (20\%).

potentially leading to implementation problems. Thus, the design was modified leading to the present value. If one wants to attenuate this overshoot further, a higher penalty on the control input must be used and the performance requirements on the output must be relaxed accordingly. Alternatively, the peak control action can be substantially reduced by changing setpoints at a constant speed (a common industrial practice), rather than using an abrupt step change.

When the operating point moves to $R_{o}=45 \Omega$, the settling times are about doubled and the overshoot of the output response is about $10 \%$, while the overshoot of the control action corresponding to a step disturbance at the input (modeling a sudden drop of the line voltage) decreases. This is mainly due to the significant decrease in plant static gain (see Fig. 3). The $\mu$-controller is in fact undertuned for this operating point, in order to get an overall good performance. When the operating point moves towards heavier loads, the responses are almost the same as the nominal, with the exception of the appearance of some chattering in both the output and the control input, for the case $R_{o}=13 \Omega$ (note that this load is beyond the range considered in our design). The occurrence of chattering is due the larger peak in the plant frequency response as the load increases. It can be eliminated by increasing the damping of the system.

Note that the controller achieves robust performance, since the performance specifications are met at all operating points. However significant variation of performance is observed. This is a direct result of the large variation in the plant dynamics, and any fixed linear controller can do very little in this respect. Reducing this variation will necessitate using a nonlinear, gain scheduling controller. 

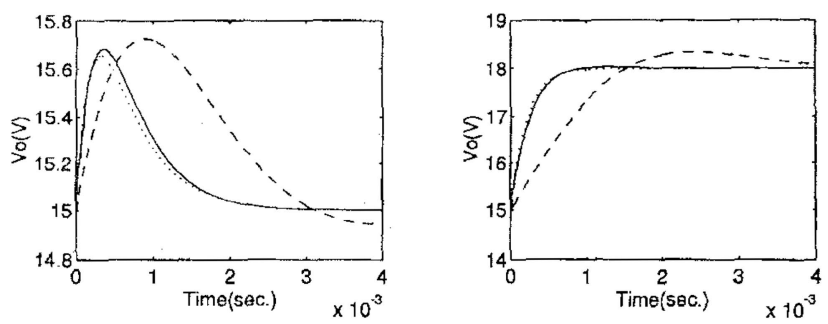

(a)
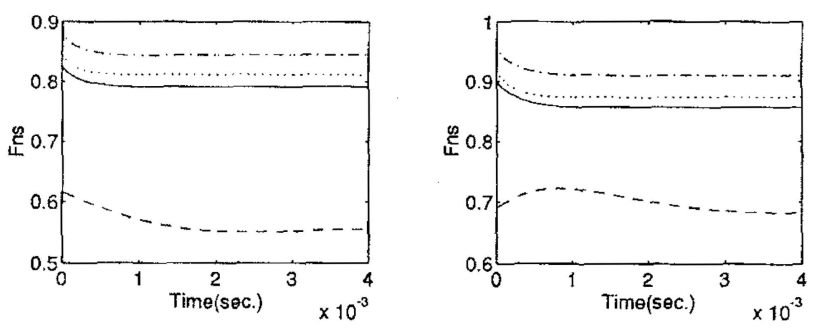

(b)

Fig. 10. Linear simulation results with PI controller at different operating points $R_{o}=19$ (solid), 45 (dash), 17 (dot), 13 (dot-dash) $\Omega$. (a) Line voltage step change $(20 \%)$.

(b) Reference input step change (20\%).

The same simulation was performed for the closed-loop system corresponding to the PI controller. The time responses to $20 \%$ step change in line voltage $V_{n g}$ and reference input $r$ at three different operating points: $R_{o}=45 \Omega, 19 \Omega$ and $17 \Omega$ are shown in Fig. 10 . They are similar to the responses with the $\mu$-controller with a slightly larger overshoot and slower responses. However, the system becomes unstable for $R_{o} \leq 15 \Omega$. This is due to the PI controller inability to provide enough attenuation to counteract the increment in the magnitude of the resonant peak of the plant at heavier loads. Since this peak increases very fast, a slight extension of operating range to heavier load requires a substantial decrease in the gain of the PI controller, seriously compromising its performance.

\section{B. Nonlinear Simulation}

It should be noted that in Section VA only linear simulations at different load conditions were used to estimate the robustness of the feedback system and the load regulation performance. While linear simulations at different load conditions often can provide and approximate evaluation of load regulation performance, this is usually insufficient to assess the performance of a highly nonlinear system such as the converter. Thus, to further validate our results, a nonlinear simulation of the SRC circuit was performed using P-Spice. The closed-loop system was obtained by first realizing the transfer function (21) using operational amplifiers and then connecting this controller to the converter.
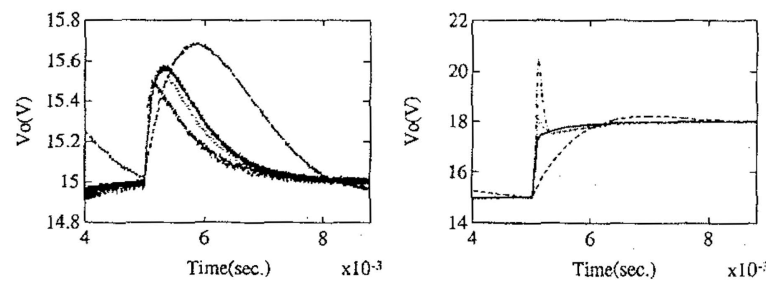

(a)
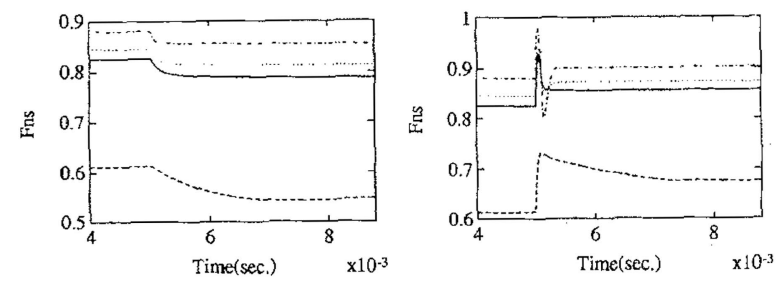

(b)

Fig. 11. Nonlinear simulation results with $\mu$-controller at different operating points $R_{o}=19$ (solid), 45 (dash), 17 (dot), 13 (dot-dash) $\Omega$. (a) Line voltage step change (20\%). (b) Reference input step change (20\%).
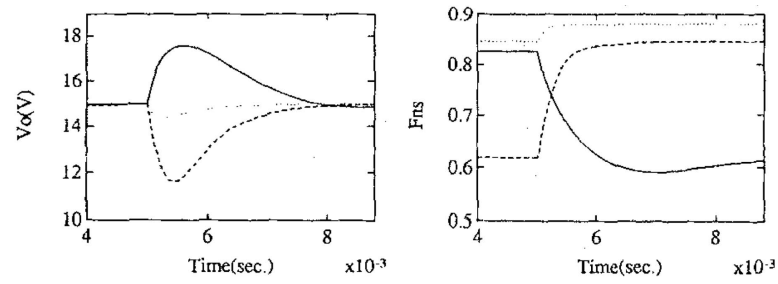

Fig. 12. Nonlinear simulation of load regulations. Load step changes from $19 \Omega$ to $45 \Omega$ (solid), from $45 \Omega$ to $17 \Omega$ (dash), from $17 \Omega$ to $13 \Omega$ (dot).

Fig. 11 shows the responses due to reference input and line voltage step changes. Note that these results are similar to those obtained using a linear simulation as shown in Fig. 9. In the responses to line voltage step change, both the overshoot and settling time are slightly larger than in the linear simulation. In the responses to reference input step change, the overshoot increases very fast as the load increases and it becomes unacceptable at the operating point $R_{o}=13 \Omega$. (Note however that this load is not covered in our control design). The chattering observed in the responses to a line voltage step change is fundamentally different from that appearing in the linear simulation. This chattering is due to the periodic behavior of the converter (periodic charge and discharge of capacitor) and has nothing to do with underdamping. Of course similar phenomena occur in the responses to a reference input step change, but the ripples are too small to be visible compared with the scale used there.

Fig. 12 shows the load regulation characteristics for three cases: a load $R_{o}$ step change from $19 \Omega$ to $45 \Omega$, from $45 \Omega$ to $17 \Omega$, and from $17 \Omega$ to $13 \Omega$. These plots 
show that the resulting closed-loop system has both good performance and good robustness characteristics.

\section{CONCLUSIONS}

Because of the ever increasing demand for smaller size and lighter weight, resonant dc-to-dc converters have been the object of much attention lately. These converters have the potential to provide high-performance conversion, without some of the problems associated with classical PWM-based converters, provided that a suitable control circuit, guaranteeing performance in the face of line-input disturbances, load changes, and component variations can be synthesized.

In this paper we have studied these problems within the framework of $\mu$-synthesis. In order to cast our problem into this framework, uncertainties in the load and components are modeled as a single, norm-bound, complex perturbation covering all possible plants. The design example of Section $\mathrm{V}$ demonstrates that different performance requirements can be easily incorporated by using suitable weights on the corresponding input and output signals and that conflicting performance specifications can be traded-off by adjusting these weights. The simulation results show that the resulting controller fully satisfies the design objectives. These results are further validated through a full nonlinear circuit simulation.

The paper shows that the $\mu$-robust control framework provides a systematic way for synthesizing controllers for resonant converters, capable of guaranteeing good performance under a wide range of load conditions. By lumping uncertainties from different sources into one perturbation block, the resulting robust performance problem has only two uncertainty blocks. Hence the upper bound of $\mu$ used in the synthesis process coincides with the exact value.

\section{REFERENCES}

[1] Balas, G. J., et al. (1993) $\mu$-Analysis and synthesis toolbox, version 2.0. MUSYN Inc. and The MathWorks, Inc., June 1993.

[2] Batarseh, I. (1990) Steady state analysis and design of high order resonant converters.

Ph.D. dissertation, University of Illinois-Chicago, June 1990.

[3] Batarseh, I., and Lee, C. Q. (1991) Steady-state analysis of the parallel resonant converter with LLCC-type commutation network.

IEEE Transactions on Power Electronics, 6, 3 (July 1991), 525-538.

[4] Batarseh, I., and Severns, R. Resonant converter topologies with three and four energy storage elements.

To be published in IEEE-Transactions on Power Electronics.
[5] Bhat, A. S., and Dewan, S. B. (1986)

A generalized approach for the steady state analysis of resonant converters.

In Proceedings of IEEE Annual Power Electronics Conference (APEC'86), (1986), 664-671.

[6] Chiang, R. Y., and Safonov, M. G. (1992)

Robust control toolbox, version 2.0.

The MathWorks, Inc., Aug. 1992.

[7] Doyle, J. C. (1982)

Analysis of feedback systems with structured uncertainties. IEE Proceedings, Pt. D, 129, 6 (1982), 242-250.

[8] Doyle, J. C. (1983)

Synthesis of robust controllers and filters. In Proceedings of IEEE Conference on Decision and Control, San Antonio, TX, Dec. 14-16, 1983.

[9] Franklin, G., Powell, D., and Workman, M. (1990)

Digital Control of Dynamic Systems.

Reading, MA: Addison Wesley, 1990.

[10] Freeland, S. (1988)

An introduction to the principle and features of resonant power conversion.

Rockwell International Corporation-Autonetics ICBM Systems Division, Anaheim, CA, 1988.

[11] Johnson, S., and Erickson, R. W. (1986)

Steady-state analysis and design of the parallel resonant converters.

In Proceedings of IEEE Power Electronics Specialists (PESC'86), 1986, 154-165.

[12] Kassakian, J., and Schlecht, M. (1988)

High frequency high density converters for distributed power supply systems.

Proceedings of the IEEE, 76, 4 (Apr. 1988).

[13] King, R., and Stuart, T. (1983)

Inhereted overload protection for the series resonant converter.

IEEE Transactions on Aerospace and Electronic Systems, (Nov. 1983), 820-830.

[14] Lee, C. Q., and Batarseh, I. (1987)

Performance characteristics of parallel resonant converter. IEE Electronics Letters, 23 (Nov. 1987), 1273-1274.

[15] Liu, K., Oruganti, R., and Lee, F. C. (1987) Quasi-resonant converters: Topologies and characteristics. IEEE Transactions on Power Electronics, 2, 1 (1987), 62-71.

[16] Lundstrom, P., Skogestad, S., and Wang, Z.-Q. (1991)

Performance weight selection for $H$-infinity and $\mu$-control method.

Transactions of the Institute of Measurement and Control, 13, 5 (1991), 241-252.

[17] Lundstrom, P., Skogestad, S., and Wang, Z.-Q. (1991)

Uncertainty weight selection for $H$-infinity and $\mu$-control methods.

In Proceedings of IEEE Conference on Decision Control, Brighton, Dec. 1991.

[18] Middlebrook, R. D., and Cuk, S. (1981)

Advances in Switched-Mode Power Conversion, Vols. 1, 2 3.

TESLACO, Pasadena, CA, 1981.

[19] Oruganti, R. (1987)

State-plane analysis of resonant converters.

$\mathrm{Ph} . \mathrm{D}$. dissertation, Virginia Polytechnic Institute and State University, Blackburg, Aug. 1987.

[20] Oruganti, R., and Lee, F. C. (1985)

State-plane analysis of parallel resonant converter. In Proceedings of IEEE Power Electronics Specialists Conference (PESC'85), (1985), 56-73.

[21] Oruganti, R., and Lee, F. C. (1985)

Resonant power processing: Part I. State-plane analysis. IEEE Transactions on Industrial Application, (Nov./Dec. 1985), 1453-1460. 
Resonant power processing: Part II. Methods of control. IEEE Transactions on Industrial Application, (Nov./Dec. 1985), 1461-1471.

[23] Packard, A., and Doyle, J. C. (1993)

The complex structured singular value. Automatica, 29, 1 (1993), 71-109.

[24] Packard, A., Doyle, J. C., and Balas, G. (1993)

Linear, multivariable robust control with a $\mu$ perspective. Transactions of the American Society of Machanical Engineers, 115 (June 1993), 426-437.

[25] Safonov, M. G. (1982)

Stability margins of diagonally perturbed multivariable feedback systems.

IEE Proceedings, Pt. D, 129, 6 (1982), 251-255.

[26] Safonov, M. G. (1983)

$L_{\infty}$ optimization vs. stability margins.

In Proceedings of the IEEE Conference on Decision and Control, San Antonio, TX, Dec. 14-16, 1983.

[27] Safonov, M. G., and Athans, M. (1981)

A multiloop generalization of the circle criterion for stability margin analysis.

IEEE Transactions on Automatic Control, AC-26, 2 (1981), 415-422.

[28] Siri, K., and Lee, C. Q. (1986)

Design of series resonant converter with normalized state-plane diagram.

IEEE Transactions on Aerospace and Electronic Systems, 22, 6 (1986), 757-763.

[29] Siri, K., and Lee, C. Q. (1991)

State-plane approach to frequency response of resonant converters.

Proceedings of IEE, Pt. G, Electronic Circuits and Systems, 138, 3 (Oct. 1991), 557-563.
[30] Skogestad, S., Morari, M., and Doyle, J. C. (1988)

Robust control of ill-conditioned plants: High-purity distillation.

IEEE Transactions on Automatic Control, AC-33, 12 (1988), 1092-1105.

[31] Steigerwald, R. L. (1988)

A comparison of half-bridge resonant converter topologies.

IEEE Transactions on Power Electronics, 3, 2 (1988), 174-182.

[32] Sznaier, M. (1994)

Mixed $l^{1} / \mathcal{H}_{\infty}$ controllers for SISO discrete time systems. Systems and Control Letters, 23 (Sept. 1994), 179-186.

[33] Todd, P., and Lutz, R. (1986)

A practical parallel loaded resonant power supply.

In Proceedings of IEEE Annual Power Electronics

Conference (APEC'86), (Apr. 1986), 90-97.

[34] Vorperian, V. (1984)

Analysis of resonant converters.

Ph.D. dissertation, California Institute of Technology, Pasadena, May 1984.

[35] Wang, Z.-Q., Lunderström, P., and Skogestad, S. (1994)

Representation of uncertain time delays in the $H_{\infty}$ framework

International Journal of Control (special issue on advances in process control), 59, 3 (1994), 627-638.

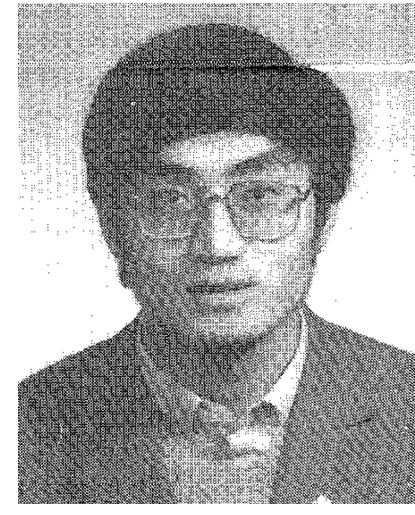

Zi-Qin Wang was born in China on January 13, 1963. He received the B.S. degree in power engineering from the Wuhan Institute of Hydraulic and Electronic Engineering in 1983, and the M.S. and Ph.D. degrees in control engineering from the Southeast University (formerly Nanjing Institute of Technology) in 1986 and 1990, respectively.

He was a faculty member at the Southeast University and a Norwegian Research Fellow at the Norwegian Institute of Technology, Trondheim. He is presently a postdoctoral student at the Pennsylvania State University, University Park. His research interests include robust and nonlinear control design, neural network for control, discrete event dynamic systems, and industrial applications. 

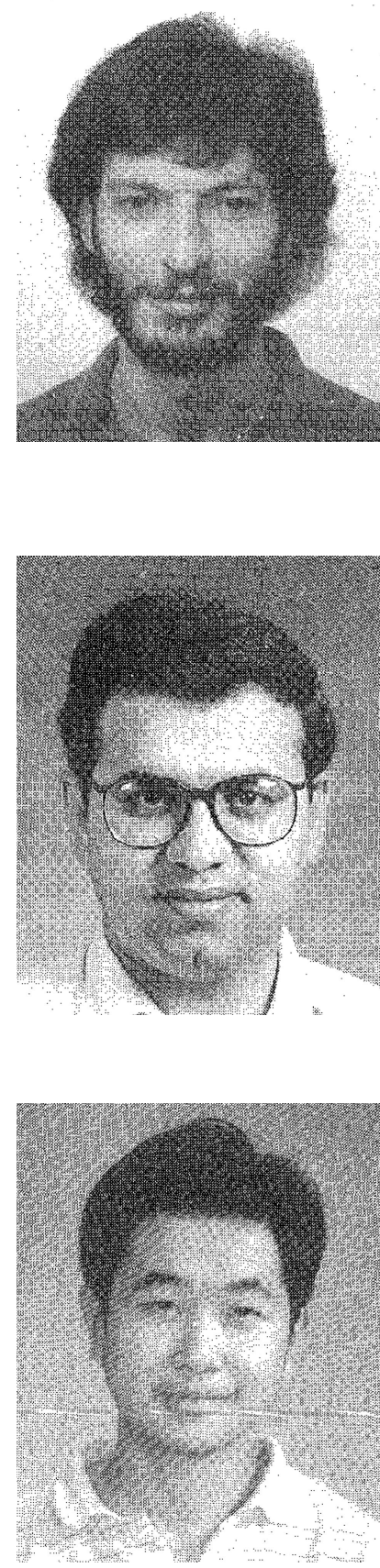

Mario Sznaier (M'89) received the Ingeniero Electronico and Ingeniero en Sistemas de Computacion degrees from the Universidad de la Republica, Uruguay in 1983 and 1984, respectively, and the MSEE and Ph.D. degrees from the University of Washington, Seattle, in 1986 and 1989, respectively.

He spent the year 1990 as a Research Fellow in Electrical Engineering at the California Institute of Technology, Pasadena. From 1991 to 1993 he was an Assistant Professor of Electrical Engineering at the University of Central Florida, Orlando. In 1993 he joined the Department of Electrical Engineering at the Pennsylvania State University, University Park, where he is currently an Assistant Professor. His research interest include multiobjective robust control, applications of robust control to power electronics, and robustness and stability issues in intelligent control.

In 1992 Dr. Sznaier was awarded a National Science Foundation Research Initiation Award for his research on robust control of systems under mixed time/frequency domain performance specifications. He is a member of SIAM, Tau Beta Pi, and Eta Kappa Nu. From 1992 to 1993 he served as the faculty advisor to the IEEE Student Branch at UCF, and since 1993 he has been a co-advisor to Penn State's IEEE Student Branch. He served in the program committee of the 1994 IEEE CDC and is a member of the Control Systems Society Conference Editorial Board.

Issa Batarseh (S'83-M'91-SM'92) was born in Jordan on January 29, 1961. He received his B.S., M.S. and Ph.D. from the University of Illinois at Chicago in 1983, 1985, and 1990, respectively, all in electrical engineering.

He was a visiting Assistant Professor in the Electrical Engineering Department at Purdue University Calumet, from 1989 to 1990. In August 1991, he joined the Department of Electrical and Computer Engineering at the University of Central Florida in Orlando, as an Assistant Professor. His research interests include PWM and high frequency resonant converters, power factor correction circuits and small signal modeling of dc-to-dc resonant converters.

Dr. Batarseh is a member of Tau Beta Pi and Eta Kappa Nu and is presently serving as an advisor to Eta Kappa $\mathrm{Nu}$ at the University of Central Florida. He is a registered Professional Engineer in Florida. He has served on the program committees of IEEE, APEC, PESC, and IAS.

Juanyu Bu was born in China on July 4, 1974. He received the B.S. degree in automatic control from the University of Science and Technology of China in 1992, and the M.S. degree in electrical engineering in 1994 from the Pennsylvania State University, University Park, where he is currently working towards the Ph.D. degree.

He has served as a research assistant in the Control and System Lab. His research interests include robust and optimal control design, performance analysis and simulations. 